\title{
Design of the mobile-robot-based surveillance system on university campuses to reduce the effects of COVID-19 pandemic
}

\author{
Quy Xuan Dao \\ Eastern International University \\ quy.dao@eiu.edu.vn
}

\author{
Viet Thanh Cao \\ Eastern International University \\ viet.cao@eiu.edu.vn \\ Linh Thi Kim Linh \\ Eastern International University \\ linh.ngo@eiu.edu.vn
}

\author{
Duc Ngoc Trinh \\ Eastern International University \\ duc.trinh@eiu.edu.vn
}

\begin{abstract}
This paper introduces a new surveillance system to detect wearing-mask and monitor social distancing and body temperature to reduce the effects of the COVID-19 pandemic on university campuses. This surveillance system was designed and implemented to SunBot, an autonomous mobile, based on hardware including Jetson Nano, camera, and thermal camera, and open-source software including OpenCV, YOLOv3, MobilNetv2, TensorFlow, Keras. Both hardware and software are basic, simple to deploy, and affordable cost. Experimental results showed that the surveillance system deployed on university campuses to reduce the effects of the COVID-19 pandemic worked as expected.
\end{abstract}

Index Terms-mask detection, social distancing monitoring, body temperature monitoring, covid-19

\section{INTRODUCTION}

W E CURRENTLY face the COVID-19 pandemic due to the agent SARS-COV-2 virus which is recognized as a global pandemic by the World Health Organization [1] because of its effects and damages. The COVID-19 virus can be fatal because it damages the respiratory human [2]. As of December 15,2020 , the COVID-19 pandemic has been crossing over 200 countries in the world, there were $73,143,329$ cases, $1,627,046$ deaths, 51,263,541 cures [3]. In which, the United States is the most affected country with $16,909,295$ cases, 307,853 deaths, $9,828,401$ cures. Everyone is affected and has a high risk of death [2]. In the UNESCO report [4], the COVID-19 pandemic has affected billions of students, and thousands of schools. According to UNESCO monitoring, 1,576,021,818 learners are currently affected due to school closures in response to the pandemic, impacting about $91.3 \%$ of the total number of learners in 188 countries. In order to reduce the spread of COVID-19, the government decided to temporarily close educational institutions. But this solution is only according to a short-term solution. Teaching and learning must continue when the vaccine is currently developed and tested.

This paper aims to highlight the benefits of the surveillance system in fighting COVID-19 on university campuses. The
World Health Organization recommended people should wear a face mask in public areas because it is one of the effective protection methods since the COVID-19 pandemic becomes a global health crisis. In order to determine wearing a mask, the authors [5] proposed a face mask detection model based on a hybrid model using deep and classical machine learning including two components: (1) Resnet50; (2) Decision trees, Support Vector Machine (SVM). This method achieved more than $99 \%$ testing accuracy. Similarly to [5], the authors [6] proposed mask detection based on YOLOv2 network with ResNet-50. This result showed the highest average precision percentage of $81 \%$ by using the adam optimizer. In [7], the author presented a simplified approach to performing mask detection using the basic Machine Learning (ML) packages such as TensorFlow, Keras, OpenCV, and Scikit-Learn.

Another author [8] deployed an IoT-based solution that used Arduino Uno to perform body temperature checks based on an infrared sensor or thermal camera, and Raspberry Pi to perform mask detection and social distancing check. In [9], the authors proposed a B5G framework to develop a mass surveillance system to monitor social distancing, mask-wearing, and body temperature based on three deep learning models including ResNet50, Deep tree, and Inceptionv3. In another research, the author [10] presented a method to determine the social distance between two people by using a camera placed on a mobile robot to estimate the distance. The robot was also equipped with a thermal camera to monitor body temperature.

In [11], the author proposed use of the technologies such as IoT, UAVs, Blockchain, AI, and $5 \mathrm{G}$ in order to reduce the impact of the COVID-19 pandemic. More detail of style and technology robots in the COVID-19 pandemic, Murply et al. [12] presented the application of robots for COVID19 response by summarizing 262 reports appearing between March 27 and July 4, 2020. These reports described 203 instances of the actual use of 104 different robot models for the COVID-19 response. In 203 instances, public safety was the largest number of the reported instance (74), clinical care (46), 


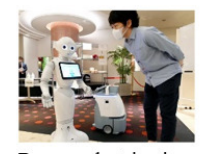

Peper robot in time of the COVID-19 pandemic

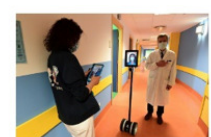

Hospital communicaCOVID-19 pandemic viole

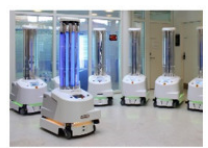

Virus killing ultra MiR delivering hospiviolet-disinfection tal equipment robot
Fig. 1: Robots in the COVID-19 time ( [14]).

quality of life (27), continuity of work and education (22/203), laboratory and supply chain automation (21), and non-clinical care (13). To control robots, teleoperation, automation, and autonomy were reported as 105,74 , and 24 , respectively. In other research, the author [13] summarized some exciting and important new research on mobile robots which is deployed in the COVID-19 pandemic time. As shown in Fig. 1, the author [14] presented some different applications of mobile robots in order to fight the COVID-19 pandemic such as population awareness and control, delivery services, disinfection of facilities, transport of material and supplies, etc. In another research, the author [15] concerned about how robotic and autonomous systems and smart wearable complement and support healthcare delivery and the healthcare staff during the COVID-19 pandemic.

Leveraging artificial intelligence, especially deep learning, the implementation of the surveillance system is becoming simpler and more effective. It is possible to implement this system using the available hardware such as Arduino, Raspberry $\mathrm{Pi}$, and Jetson Nano and open-source software including machine learning libraries such as OpenCV, YOLOv3, MobileNetv2, TensorFlow, Keras, etc. To continue learning as well as ensure social distancing, a surveillance system to detect mask-wearing and monitor social distancing and body temperature are expected to deploy on university campuses. In this paper, we present a surveillance system implemented into SunBot (multiple functional mobile robots based on Jetson Nano, depth camera, and thermal camera) that serve as a healthcare assistant to detect mask-wearing and monitor social distancing and body temperature.

This paper is organized as follows: Section 2 describes the SunBot surveillance system that we consider healthcare functions including mask detection, social distancing monitoring, and body temperature monitoring, Section 3 shows the experimental results when we deployed SunBot surveillance system on the Eastern International University (EIU) campus during the social distancing time, and Section 4 are some conclusion as well as future work.

\section{SunBot Surveillance System}

This section presents SunBot surveillance system to detect wearing-mask as well as monitor social distancing and body temperature. SunBot was designed and illustrated in Fig. 2. SunBot was designed to perform five functions such as (1) delivery, (2) telepresence, (3) guide, (4) healthcare, (5) security, (6) mask detection and (7) social distancing monitoring. The proposed mobile robot contains two main parts: platform

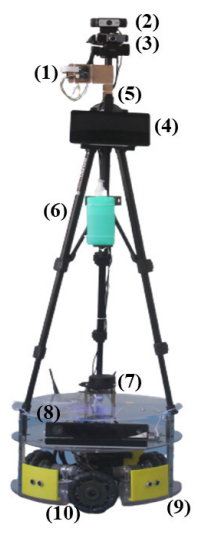

Fig. 2: Full optional SunBot:(1) thermal camera, (2) - (3) cameras, (4) tablet/screen, (5) speaker/mic, (6) hand clean bottle, (7) lidar, (8) depth camera, (9) ultra-sonic senor and (10) Omni wheels.

and tool. Jetson Nano and Microcontroller STM32F4 are used to program and operate the mobile robot based on the ROS framework. The sensors, cameras, and lidar are chosen in order to provide information that helps the mobile robot to move in autonomy. Jet-son Nano, Raspberry Pi, camera and thermal camera perform mask detection, social distancing, and body temperature monitoring based on open-source such as OpenCV, MobileNetv2 [16], YOLOv3 [17], TensorFlow, Keras, etc. In this paper, we only focus on mask detection and social distancing monitoring of SunBot.

\section{A. Autonomous Mobile Module}

This module allows SunBot to move on university campuses in autonomy. This module was built based on selfdriving-cars technology including computer vision and sensor technologies. We built SunBot platform based on open-source hardware and software: TurtleBot2 [18] and self-driving-cars technology using a convolution neural network (CNN) [19]. We also built Information and Communications Technology (ICT) infrastructure on the EIU campus to support SunBot. Fig. 3 shows hardware and software requirements to build this module. Jetson Nano, Lidar, and RGB-D camera are important hardware while ROS, OpenCV, and CNN are required software. Information from Lidar and RGB-D camera are processed by Jetson Nano based on CNN [19] to control mobile robots. Two sub-modules are navigation (ROS) and avoidance $(\mathrm{OpenCV}+\mathrm{CNN})$ that help SunBot to move around university campuses.

\section{B. Virtual Assistant Module}

This module allows SunBot to communicate with users based on natural language processing. SunBot can communicate with users by speech or text. In our previous work [20], [21] we developed a virtual assistant for online learning. This system was implemented into SunBot to communicate with users based on healthcare functions. Our virtual 


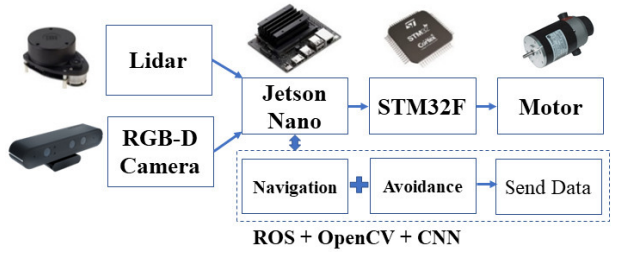

Fig. 3: Hardware and Software for Autonomous Mobile Module.

assistant is a hybrid method that is a combination of rulebased chatbots and AI-based chatbots. Our virtual assistant was developed based on Rasa's "Open-source machine learning framework to automate text- and voice-based conversations" [22]. Fig. 4 shows hardware and software requirements to build the virtual assistant module. We use Jetson Nano, Camera, Speaker, and Monitor as hardware while OpenCV and Google Text-to-Speech (T2S)/Speech-to-Text (S2T) API as software. The face/speech detection module allows SunBot to recognize users while natural language processing helps SunBot to communicate with users via speech and/or text.

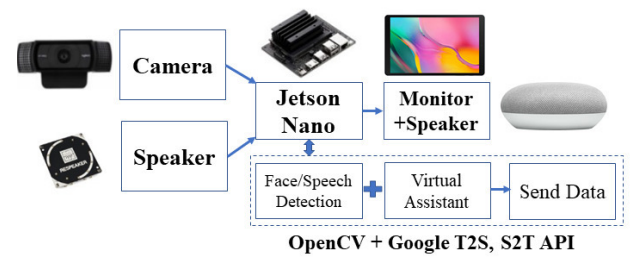

Fig. 4: Hardware and Software for Virtual Assistant Module.

\section{Mask Detection Module}

During the COVID-19 time, wearing-mask becomes an important way to fight the COVID-19 pandemic. SunBot surveillance system was designed to detect wearing-mask by using OpenCV, and MobileNetv2 [16]. This is one of the simplest ways to detect wearing-mask. There are many ways to detect wearing-mask but the one that we used because of its simplicity and efficiency and accepted price. Fig. 5 shows hardware and software requirements. Hardware includes Jetson Nano, camera, monitor, and speaker while software includes OpenCV, TensorFlow, Keras, and MobileNetv2. The Mask detection module allows us to distinguish whether to wear a mask or not. This function is very important in deploying SunBot surveillance system on university campuses. Furthermore, SunBot surveillance system reminds students to wear a mask on university campuses via virtual assistant module by speech.

\section{Social Distancing Monitoring Module}

To practice social distancing or estimate the number of the people [23], we need to stay at least 6 feet from other people. The social distancing monitoring module was built based on hardware such as Jetson Nano, camera, monitor, speaker, and software such as OpenCV and YOLOv3 [17]

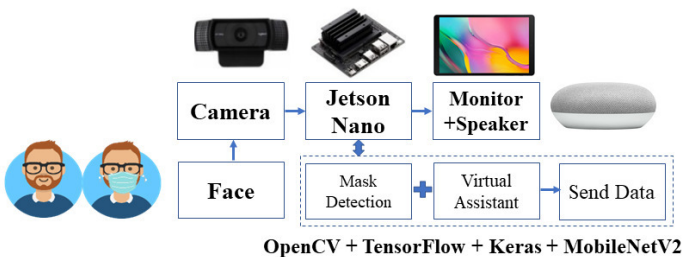

Fig. 5: Hardware and Software for Mask Detection Module.

illustrated in Fig. 6. Universities need to keep social distancing during the COVID-19 time. We estimate a distance between two people by using OpenCV and YOLOv3. We introduce one of the simplest methods to determine social distancing. People are recognized and label by rectangle boxes. The Euclidean distance of the center of these rectangle boxes is considered social distancing. When the social distancing is less than a setup threshold, SunBot surveillance system reminds students as well as requires students to keep a social distancing through three levels of distance as (1) high risk if the distance between two peoples is less than the set-up threshold (ex. within about 6 feet), (2) safe if the distance between two peoples is greater than the setup threshold (ex. within about 6-12 feet), and (3) low risk if the distance between two peoples is large enough (ex. from 12 feet).

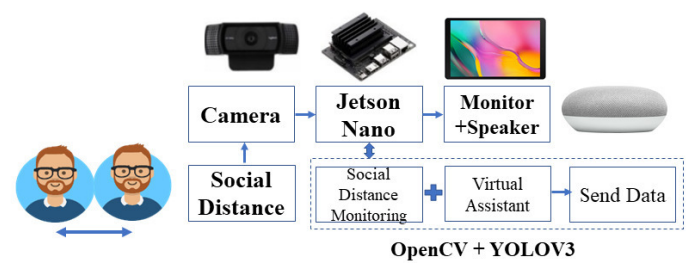

Fig. 6: Hardware and Software for Social Distancing Monitoring Module.

\section{E. Body Temperature Monitoring Module}

One of the most common and recognizable symptoms of covid-19 is a fever. Therefore, we need to check body temperature to perform the COVID-19 screening. The COVID-19 pandemic is thought to be spread mainly through close personto-person contact. Body temperature monitoring by using a thermal camera [24] is the right selection in this situation. Fig. 7 shows hardware and software requirements. We use an MLX90640 thermal camera and Raspberry Pi as hardware and OpenCV as software to monitor body temperature. When the body temperature is greater than a setup threshold, SunBot surveillance system recognizes people at risk of disease.

\section{EXPERIMENTAL RESULTS}

SunBot surveillance system was deployed on the EIU campus to detect wearing-mask and monitor social distancing and body temperature. Sunbot moves around the EIU campus to perform surveillance functions at gates, libraries, laboratories, and cafeterias. 


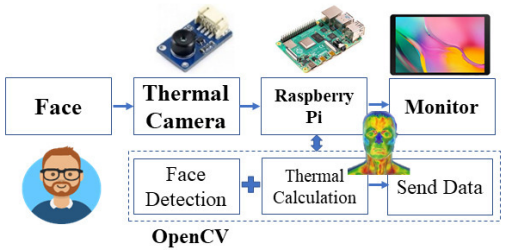

Fig. 7: Hardware and Software for Body Temperature Monitoring Module.

\section{A. Mask Detection}

First of all, we choose a dataset in [25] to train a mask detection model. Fig. 8 shows how to wear a mask in the COVID-19 pandemic. When we use this dataset (first case), we could obtain a wrong result illustrated in Fig. 9. In the first case, the training dataset is labeled "mask" containing images of wearing a mask under the nose. This dataset needs to be modified to train the mask detection model. In the second case, the result becomes better when we replace images wearing a mask under the nose with the "without a mask" dataset. Therefore, we use the modified dataset (second case) to train the mask detection model.
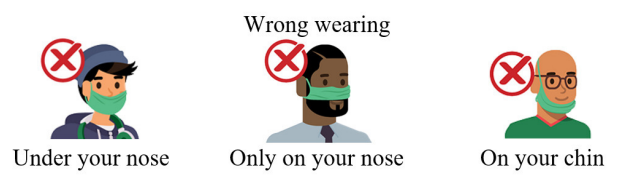

Right wearing

Fig. 8: How to wear a mask.

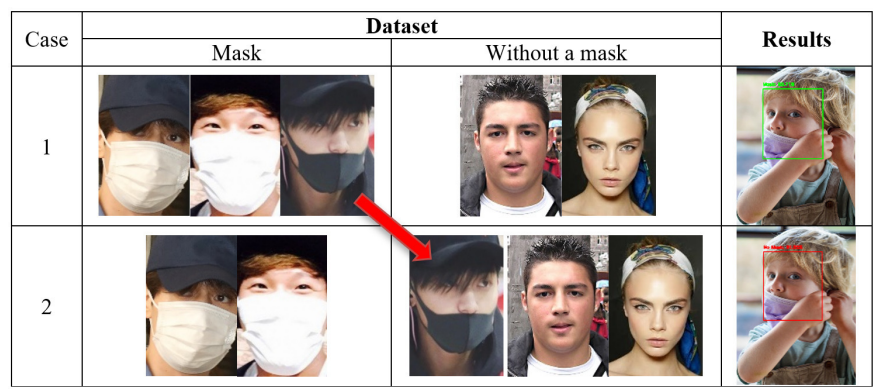

Fig. 9: Dataset for Mask Detection.

We implemented the mask detection model above to Jetson Nano and camera to detect wearing-mask on the EIU campus. The scenario of mask detection is given as (1) SunBot moves around the EIU campus to detect wearing-mask and capture video; (2) SunBot surveillance system reminds "without mask" cases; (3) SunBot sends information to cloud-management center to store data. Since a high proportion of people comply with wearing-mask that have a substantial impact on COVID19 transmission on campus, SunBot statistics the number of people who do not wear masks to notify the administrative center. Fig. 10 shows experimental results for mask detection task on the EIU campus. Although people wear different masks from medical masks to cloth masks, SunBot surveillance system can distinguish between people who wear masks or not.

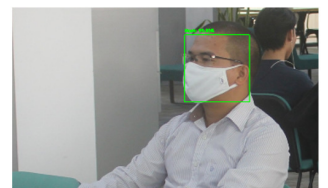

(a) Mask

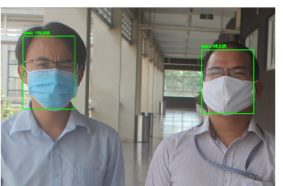

(b) Mask

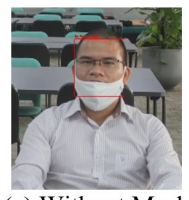

(c) Without Mask
Fig. 10: Mask Detection on the EIU campus.

\section{B. Social Distancing Monitoring}

Universities need to keep social distancing between individuals in order to reduce transmission of the COVID-19 spread. SunBot moves around university campuses to ensure everyone that must consistently follow social distancing practices. We implemented the social distancing monitoring model based on YOLOv3 to Jetson Nano and camera to determine social distancing measurement on the EIU campus. Fig. 11 and 12 shows experimental results on the EIU campus. SunBot surveillance system establishes policy and reminders to keep at least 6 feet of space between individuals including three warning levels as high risk, safe, and low risk.

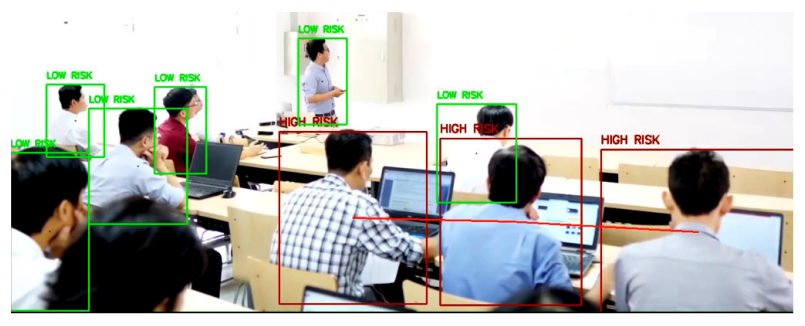

Fig. 11: Social Distancing Monitoring in Classroom.

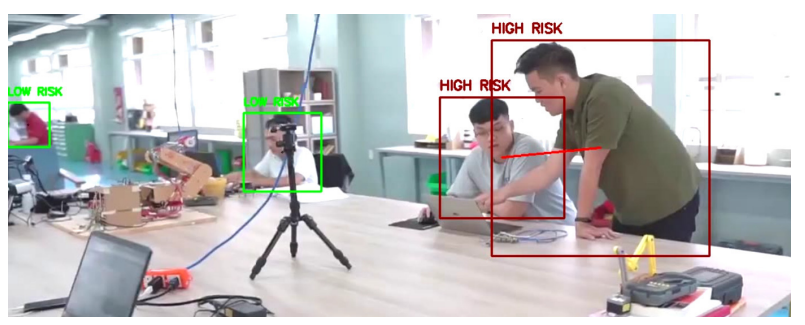

Fig. 12: Social Distancing Monitoring in Laboratory.

\section{Body Temperature Monitoring}

Fig. 13 illustrates the experimental result of body temperature monitoring based on the MLX90640 thermal camera which captures temperature from $-40^{\circ} \mathrm{C}$ to $300^{\circ} \mathrm{C}$. We easily configure a body temperature monitoring alert at $37^{\circ} \mathrm{C}$. The scenario of body temperature monitoring is given as follows (1) users move before the thermal camera, (2) the thermal camera checks the users' body temperature, shows information, 
and sends data to the cloud management system, (3) SunBot communicates with users about their status and provides health advice and guidance. Furthermore, the virtual assistant also provides Questions and Answers about the COVID-19 pandemic.
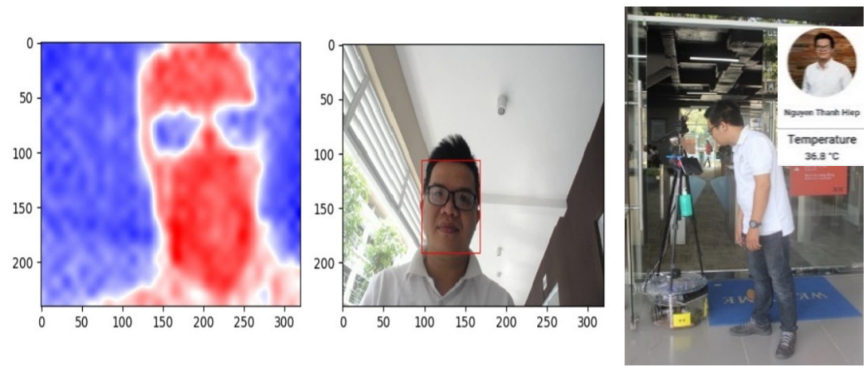

Fig. 13: Body Temperature Monitoring at $36.8^{\circ} \mathrm{C}$.

\section{Discussion}

To achieve the goal of reopening universities as safely and quickly as possible, we need to reduce the spread of COVID-19 in universities and out in the community. In the previous sections, we presented how to detect mask-wearing and monitor social distancing and body temperature on university campuses. Universities can deploy these systems and put together corresponding policies to guarantee continuing learning as (1) encourage behaviors that help reduce the spread of COVID-19, (2) maintain a healthy environment, (3) maintain healthy activities, and (4) be prepared when a coronavirus case is recognized. Students, teachers, and staff must obey fully with steps to protect themselves and others such as the correct use of masks, social distancing, and hand hygiene.

\section{CONCLUSION}

In this paper, a surveillance system including face mask detection and social distancing monitoring, and body temperature monitoring was presented. The proposed surveillance system was designed and implemented to the SunBot autonomous mobile robot. This surveillance system was developed based on open-software including OpenCV, MobileNetv2, YOLOv3, TensorFlow, Keras, and affordable hardware including Jetson Nano, camera, and thermal camera. Experimental results demonstrated that the proposed surveillance system is one of the performance ways to keep physical distancing as well as ensure continuous learning in the COVID-19 time. In the future, we will develop a complete surveillance system in small urban cities.

\section{ACKNOWLEDGEMENT}

This research was supported by Eastern International University, Binh Duong, Vietnam.

\section{REFERENCES}

[1] WHO. Who director-general's opening remarks at the media briefing on covid-19 - 11 march 2020. [Online]. Available: https://www.who.int/director-general/speeches/detail/who-directorgeneral-s-opening-remarks-at-the-media-briefing-on-covid-19-11march-2020

[2] L. Meng, F. Hua, and Z. Bian, "Coronavirus disease 2019 (covid-19): emerging and future challenges for dental and oral medicine," Journal of dental research, vol. 99, no. 5, pp. 481-487, 2020.

[3] Worldometer. Coronavirus update (live): $87,976,426$ cases and $1,898,009$ deaths from covid-19 virus pandemic - worldometer. [Online]. Available: https://www.worldometers.info/coronavirus/

[4] UNESCO. Coronavirus update (live): $87,976,426$ cases and $1,898,009$ deaths from covid-19 virus pandemic - worldometer. [Online]. Available: School closures caused by Coronavirus (Covid-19)

[5] M. Loey, G. Manogaran, M. H. N. Taha, and N. E. M. Khalifa, "A hybrid deep transfer learning model with machine learning methods for face mask detection in the era of the covid-19 pandemic," Measurement, vol. 167, p. 108288, 2021.

[6] _ - "Fighting against covid-19: A novel deep learning model based on yolo-v2 with resnet-50 for medical face mask detection," Sustainable Cities and Society, vol. 65, p. 102600, 2021.

[7] A. Das, M. W. Ansari, and R. Basak, "Covid-19 face mask detection using tensorflow, keras and opencv," in 2020 IEEE 17th India Council International Conference (INDICON). IEEE, 2020, pp. 1-5.

[8] N. Petrovic and j. v. p. y. Kocic, DJ, "Iot-based system for covid-19 indoor safety monitoring."

[9] M. S. Hossain, G. Muhammad, and N. Guizani, "Explainable ai and mass surveillance system-based healthcare framework to combat covidi9 like pandemics," IEEE Network, vol. 34, no. 4, pp. 126-132, 2020.

[10] A. J. Sathyamoorthy, U. Patel, Y. A. Savle, M. Paul, and D. Manocha, "Covid-robot: Monitoring social distancing constraints in crowded scenarios," arXiv preprint arXiv:2008.06585, 2020.

[11] V. Chamola, V. Hassija, V. Gupta, and M. Guizani, "A comprehensive review of the covid-19 pandemic and the role of iot, drones, ai, blockchain, and $5 \mathrm{~g}$ in managing its impact," Ieee access, vol. 8, pp. 90 225-90 265, 2020

[12] R. R. Murphy, V. B. M. Gandudi, and J. Adams, "Applications of robots for covid-19 response," arXiv preprint arXiv:2008.06976, 2020.

[13] D. Feil-Seifer, K. S. Haring, S. Rossi, A. R. Wagner, and T. Williams, "Where to next? the impact of covid-19 on human-robot interaction research," 2020.

[14] M. Cardona, F. Cortez, A. Palacios, and K. Cerros, "Mobile robots application against covid-19 pandemic," in 2020 IEEE ANDESCON. IEEE, 2020, pp. 1-5.

[15] M. Tavakoli, J. Carriere, and A. Torabi, "Robotics, smart wearable technologies, and autonomous intelligent systems for healthcare during the covid-19 pandemic: An analysis of the state of the art and future vision," Advanced Intelligent Systems, vol. 2, no. 7, p. 2000071, 2020.

[16] M. Sandler, A. Howard, M. Zhu, A. Zhmoginov, and L.-C. Chen, "Mobilenetv2: Inverted residuals and linear bottlenecks," in Proceedings of the IEEE conference on computer vision and pattern recognition, 2018, pp. 4510-4520.

[17] J. Redmon and A. Farhadi, "Yolov3: An incremental improvement," arXiv preprint arXiv:1804.02767, 2018.

[18] ROS-Fundation. Turtlebot2. [Online]. Available: https://www.turtlebot.com/turtlebot2/

[19] M. Bojarski, D. Del Testa, D. Dworakowski, B. Firner, B. Flepp, P. Goyal, L. D. Jackel, M. Monfort, U. Muller, J. Zhang et al., "End to end learning for self-driving cars," arXiv preprint arXiv:1604.07316, 2016.

[20] T.-M.-T. Nguyen, T.-H. Diep, B.-N. Bac, N.-B. Le, and X.-Q. Dao, "Design of online learning platform with vietnamese virtual assistant,' in 2021 6th International Conference on Intelligent Information Technology, 2021, pp. 51-57.

[21] X.-Q. Dao, N.-B. Le, and T.-M.-T. Nguyen, "Ai-powered moocs: Video lecture generation," in 2021 3rd International Conference on Image, Video and Signal Processing, 2021, pp. 95-102.

[22] RasaHQ. Open source machine learning framework to automate textand voice-based conversations: Nlu, dialogue management, connect to slack, facebook, and more - create chatbots and voice assistants. [Online]. Available: https://github.com/RasaHQ/rasa 
[23] K. Przybylek and I. Shkroba "Crowd counting' a la bourdieu: Automated estimation of the number of people," Computer Science and Information Systems, no. 00, pp. 29-29, 2020.

[24] S. Ansari and S. Salankar, "An overview on thermal image processing." in RICE, 2017, pp. 117-120.
[25] Chandrikadeb. Face-mask-detection: Face mask detection system based on computer vision and deep learning using opencv and tensorflow/keras. [Online]. Available: https://github.com/chandrikadeb7/FaceMask-Detection 